08

\title{
Деформационное уширение и тонкая структура спектральных линий в оптических спектрах диэлектрических кристаллов, содержащих редкоземельные ионы
}

\author{
(C) Н.М. Абишев ${ }^{1}$, Э.И. Байбеков ${ }^{1}$, Б.3. Малкин ${ }^{1}$, М.Н. Попова ${ }^{2}$, \\ Д.С. Пыталев ${ }^{2}$, С.А. Климин ${ }^{2}$ \\ ${ }^{1}$ Казанский (Приволжский) фредеральный университет, \\ Казань, Россия \\ ${ }^{2}$ Институт спектроскопии РАН, \\ Троицк, Москва, Россия \\ E-mail: abishevnm@gmail.com
}

\begin{abstract}
Разработана методика расчета формы спектральных линий в оптических спектрах редкоземельных ионов в кристаллах с учетом случайных деформаций упруго анизотропной кристаллической решетки, обусловленных точечными дефектами. Функция распределения компонент тензора случайных деформаций в случае малой концентрации дефектов получена в виде обобщенного шестимерного распределения Лоренца. Параметры функции распределения представлены интегральными функционалами компонент тензора деформации на сфере единичного радиуса, содержащей в центре изотропный точечный дефект. Выполнены численные расчеты тензоров деформаций, индуцированных точечными дефектами, и параметров функций распределения случайных деформаций в кристаллах $\mathrm{LiLuF}_{4}$ и $\mathrm{LaAlO}_{3}$. Вычисленная огибающая с дублетной структурой, отвечающая синглет-дублетному переходу $\Gamma_{2}\left({ }^{3} H_{4}\right) \rightarrow \Gamma_{34}\left({ }^{3} H_{5}\right)$ в спектре поглощения ионов $\operatorname{Pr}^{3+}$ в $^{3}$ кристалле $\mathrm{LiLuF}_{4}$, хорошо согласуется с данными измерений.
\end{abstract}

Работа выполнена при финансовой поддержке РФФИ (грант № 17-02-00403, Н.М.А., Э.И.Б., Б.3.М.) и программы президиума РАН 1.7 „Актуальные проблемы фотоники, зондирование неоднородных сред и материалов“ (М.Н.П., Д.С.П., С.А.К.).

DOI: 10.21883/FTT.2019.05.47589.22F

\section{1. Введение}

Ширина спектральных линий оптических материалов определяет возможности их использования в качестве активных сред в лазерах и сцинтилляторах, в оптических информационных технологиях $[1,2]$. В оптических спектрах редкоземельных (Р3) ионов в кристаллах, соответствующих $f-f$-переходам, ширины наблюдаемых линий всегда существенно больше естественной ширины участвующих в переходе уровней энергии вследствие неоднородного уширения. Неоднородное уширение является результатом взаимодействия оптических центров (Р3-ионов) с полями различной природы. В частности, взаимодействие $4 f$-электронов с полем случайных деформаций, индуцированных дефектами кристаллической решетки, обусловливает квази-непрерывное распределение энергии квантовых переходов и соответствующее неоднородное уширение. Наряду с уширением, случайные деформации формируют тонкую структуру бесфононных линий в случае переходов, в которых участвуют орбитально вырожденные состояния Р3-ионов в кристаллических полях тригональной, тетрагональной и кубической симметрии [3-6]. Деформационная тонкая структура (дублетная, триплетная) наблюдалась в оптических спектрах высокого разрешения активированных РЗ-ионами кристаллов со структурами шеелита [4], эльпасолита [5], циркона [6] и ромбоэдрического перовскита [7].
Статистическая теория деформационного уширения спектральных линий в случае переходов между невырожденными состояниями оптических центров была развита Стоунхэмом [3]. Аналитическое выражение для функции распределения компонент тензора деформаций, обусловленных точечными дефектами в упруго изотропном континууме, было получено в работе [5]. Отметим, что в рамках приближения упругого континуума точечные дефекты не приводят к неоднородному всестороннему сжатию или растяжению кристаллической решетки. В работе [6] была введена функция распределения случайных деформаций, индуцированных точечными дефектами в упруго анизотропном континууме, в полном шестимерном пространстве компонент тензора деформаций на основе обобщения аналитического выражения для двумерных функций распределения.

В настоящей работе представлена методика моделирования формы спектральных линий, соответствующих $f-f$-переходам в Р3-ионах, включающая последовательное выполнение расчетов индуцированного точечным дефектом поля смещений в упруго анизотропном континууме, параметров функции распределения случайных деформаций и параметров гамильтониана взаимодействия Р3-иона с деформированной решеткой. Сравнение с наблюдаемым спектром усредненной по распределению случайных деформаций функции формы линии при фиксированных деформациях дает возможность найти 
силу дефектов при заданной их концентрации. Приведены результаты расчетов полей деформаций и параметров функций распределения случайных деформаций в кристаллах $\mathrm{LiLuF}_{4}$ и $\mathrm{LaAlO}_{3}$. Построенный алгоритм вычислений использован в моделировании измеренной в настоящей работе линии синглет - дублетного перехода с индуцированной случайными деформациями дублетной структурой в спектре примесных ионов празеодима в кристалле $\mathrm{LiLuF}_{4}$.

\section{2. Поле деформаций, индуцированных точечным дефектом в упруго анизотропном кристалле}

Мы рассматриваем поле смещений атомов из положений равновесия в кристаллической решетке, индуцированных точечным дефектом, в рамках теории упругости в приближении анизотропного упругого континуума. Статические смещения $\mathbf{u}(\mathbf{r})$, обусловленные силами с плотностью $\mathbf{f}(\mathbf{r})$, удовлетворяют системе неоднородных дифференциальных уравнений второго порядка [8]

$$
\sum_{\beta \gamma \delta} C_{\alpha \beta \gamma \delta} \frac{\partial^{2} u_{\delta}}{\partial x_{\beta} \partial_{\gamma}}+f_{\alpha}=0,
$$

где $\mathbf{C}$ - тензор упругих постоянных среды. Плотность сил, индуцированных сферически симметричным точечным дефектом, расположенным в точке с радиусвектором $\mathbf{R}_{d}$, пропорциональна модулю всестороннего сжатия $K$ и „силе“ дефекта $\Omega$, равной изменению объема элементарной ячейки, приходящемуся на один дефект [9],

$$
\mathbf{f}(\mathbf{r})=-K \Omega \nabla \delta\left(\mathbf{r}-\mathbf{R}_{d}\right) .
$$

Методика расчета функций Грина уравнений (1) для сред различной симметрии была развита Лифшицем и Розенцвейгом [10]. Вычисление функций Грина сводится к нахождению корней алгебраического уравнения шестого порядка. Аналитические решения уравнений (1) были получены для упруго изотропного континуума и кристаллических решеток гексагональной симметрии $[10,11]$. В частности, компоненты тензора деформации $e_{\alpha \beta}=\left(\partial u_{\alpha} / \partial x_{\beta}+\partial u_{\beta} / \partial x_{\alpha}\right) / 2$, индуцированной точечным дефектом в упруго изотропном континууме, равны

$$
e_{\alpha \beta}=\frac{\Omega}{12 \pi r^{3}} \frac{1+\sigma}{1-\sigma}\left(\delta_{\alpha \beta}-\frac{3 x_{\alpha} x_{\beta}}{r^{2}}\right)
$$

где $\sigma$ - отношение Пуассона. В общем случае компоненты тензора неоднородной деформации в сферической системе координат с центром на дефекте можно представить в виде $e_{\alpha \beta}(\mathbf{r})=\pi \Omega(2 \pi r)^{-3} q_{\alpha \beta}(\theta, \varphi)$, где $r, \theta, \varphi-$ сферические координаты вектора $\mathbf{r}$, а безразмерные функции $q_{\alpha \beta}(\theta, \varphi)$ могут быть найдены с использованием численных методов, основанных на преобразовании Фурье функций Грина уравнений (1) [12-14].
$\mathrm{C}$ целью построения функции распределения случайных деформаций в тетрагональном кристалле $\mathrm{LiLuF}_{4}$ и в ромбоэдрическом кристалле $\mathrm{LaAlO}_{3}$ с пространственными группами симметрии $I 4_{1} / a$ и $R \overline{3} c$, соответственно, в настоящей работе выполнены численные расчеты функций $q_{\Gamma \lambda}(\theta, \varphi)$, определяющих линейные комбинации компонент тензора деформации $e_{\Gamma \lambda}(\mathbf{r})$, преобразующиеся по строке $\lambda$ неприводимого представления (НП) Г соответствующих фактор-групп. Расчеты были выполнены с использованием величин упругих постоянных, приведенных в статьях [15] $\left(\mathrm{LiLuF}_{4}\right)$ и [16] $\left(\mathrm{LaAlO}_{3}\right)$.

Симметризованные комбинации компонент тензора деформации в кристалле $\mathrm{LiLuF}_{4}$ были выбраны в виде $e_{1}=e\left(A_{g}^{1}\right)=\left(e_{x x}+e_{y y}+e_{z z}\right) / \sqrt{6}, e_{2}=e\left(A_{g}^{2}\right)=\left(2 e_{z z}\right.$ $\left.-e_{x x}-e_{y y}\right) / \sqrt{12}, e_{3}=e\left(B_{g}^{1}\right)=\left(e_{x x}-e_{y y}\right) / 2, e_{4}=e\left(B_{g}^{2}\right)$ $=e_{x y}, e_{5}=e\left(E_{g}, 1\right)=e_{x z}, e_{6}=e\left(E_{g}, 2\right)=e_{y z}$, где компоненты тензора деформации $e_{\alpha \beta}$ определены в кристаллографической системе координат с осью $z$ вдоль оси $c$ решетки (ось симметрии $S_{4}$ в позициях ионов $\left.\mathrm{Lu}^{3+}\right), A_{g}, B_{g}$ и $E_{g}-$ НП группы $C_{4 h}$. Здесь и далее $e(\Gamma, \lambda)=e_{\Gamma \lambda}(\mathbf{r})$ и $q(\Gamma, \lambda)=q_{\Gamma \lambda}(\theta, \varphi)$. Компоненты тензора деформации кристалла $\mathrm{LaAlO}_{3}$ мы рассматриваем также в декартовой системе координат с осью $z$ вдоль оси симметрии третьего порядка; линейные комбинации компонент тензора деформации $e_{m}(m=1-6)$, введенные выше, преобразуются по НП $A_{g}$ и $E_{g}$ точечной группы симметрии $D_{3 d}: e\left(A_{g}^{1}\right)=e_{1}, e\left(A_{g}^{2}\right)=e_{2}, e\left(E_{g}^{1}, 1\right)=e_{3}$, $e\left(E_{g}^{1}, 2\right)=e_{4}, e\left(E_{g}^{1}, 2\right)=e_{4}, e\left(E_{g}^{2}, 1\right)=e_{5}, e\left(E_{g}^{2}, 2\right)=e_{6}$.

$\mathrm{B}$ качестве примера результатов расчетов определенных выше функций сферических координат $q_{\Gamma \lambda}(\theta, \varphi)$ на рис. 1 представлены графические изображения функций $q\left(A_{g}^{1}\right)$ и $q\left(B_{g}^{2}\right)$ для кристалла $\mathrm{LiLuF}_{4}$. Неоднородные деформации всестороннего сжатия (растяжения) $e\left(A_{g}^{1}\right)$ в упруго анизотропном кристалле отличны от нуля (рис. $1, a)$, но на порядок величины меньше в сравнении с ромбическими деформациями $e\left(B_{g}^{2}\right)$ (рис. $\left.1, b\right)$. Тем не менее, полносимметричные деформации $e\left(A_{g}^{1}\right)$ дополнительно уширяют спектральные линии.

Упругая анизотропия кристаллической решетки существенно изменяет соотношения и величины компонент тензора деформации на единичной сфере. Заметим, что угловая зависимость (3) компонент тензора деформации $e_{\alpha \beta}(\mathbf{r})$ в упруго изотропном континууме описывается сферическими гармониками 2-го порядка. Аналогично, деформации $e_{\Gamma \lambda}(\mathbf{r})$, обусловленные дефектами в реальных кристаллах, могут быть разложены в ряд по сферическим гармоникам высших порядков. Как следует из выполненного нами анализа результатов вычислений, для аналитического представления численных массивов можно ограничиться в соответствующих разложениях гармониками 2-го-10-го порядков [5]. Полученные массивы данных используются ниже для нахождения параметров функций распределения случайных деформаций, индуцированных точечными дефектами. 

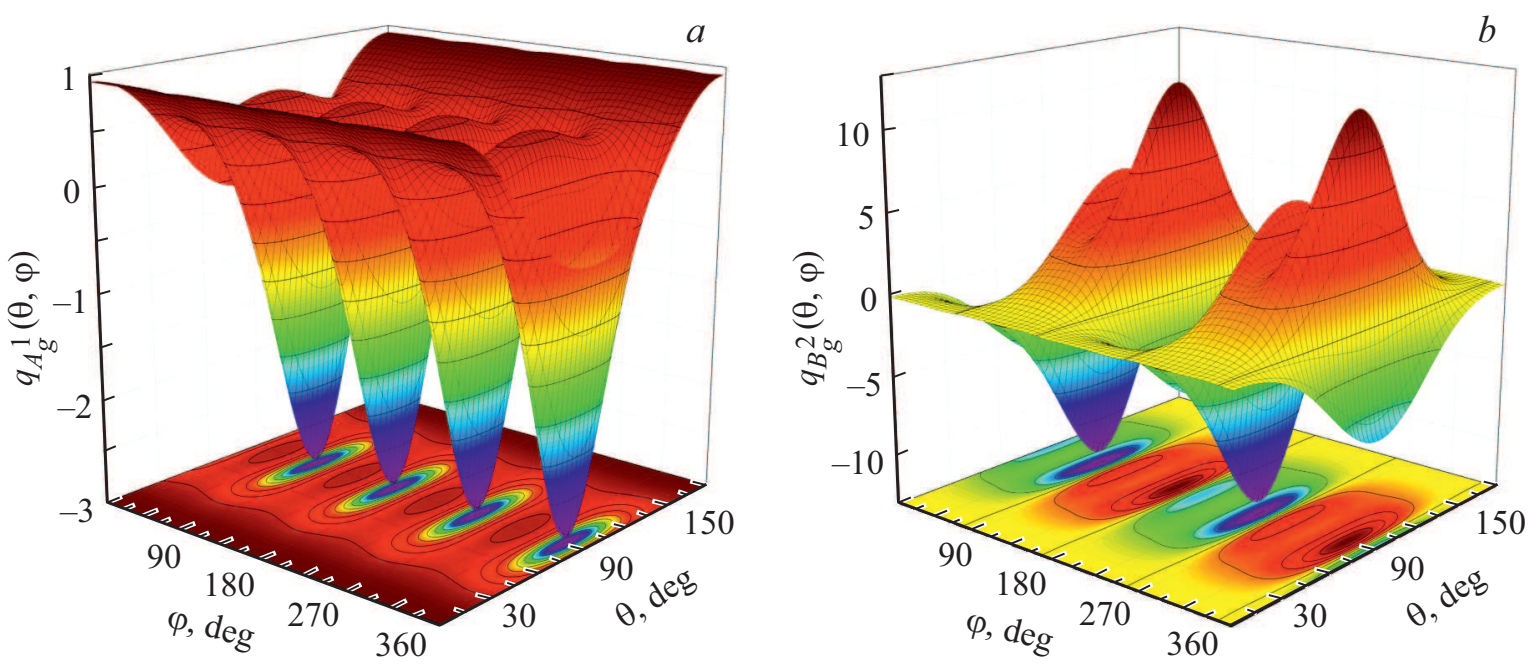

Рис. 1. Функции $q_{\Gamma \lambda}(\theta, \varphi)$, соответствующие полносимметричной $\left(a, \Gamma=A_{g}^{1}\right)$ и ромбической $\left(b, \Gamma=B_{g}^{2}\right)$ деформациям, индуцированным точечным дефектом в кристалле $\mathrm{LiLuF}_{4}$.

\section{3. Функция распределения случайных деформаций}

В рамках статистической теории функция распределения компонент тензора деформации представляется в виде

$$
g(\mathbf{e})=\left\langle\prod_{m=1}^{6} \delta\left(e_{m}-\sum_{j=1}^{N_{d}} e_{m}^{j}\right)\right\rangle
$$

где угловые скобки $\langle\ldots\rangle$ означают конфигурационное усреднение по всем возможным положениям $N_{d}$ точечных дефектов в трехмерном пространстве, $\delta(x)-$ дельта-функция Дирака, $e_{m}^{j}-$ вклад в $m$-ую компоненту тензора деформации от $j$-го дефекта. В приближении континуума и при малой концентрации точечных дефектов $C_{d}$ выражение (4) принимает вид

$$
\begin{aligned}
g(\mathbf{e})= & \frac{1}{(2 \pi)^{6}} \int_{-\infty}^{\infty} d \rho_{1} \ldots \int_{-\infty}^{\infty} d \rho_{6} \\
& \times \exp \left(-i \sum_{m=1}^{6} \rho_{m} e_{m}-C_{d} J(\boldsymbol{\rho})\right), \\
J(\boldsymbol{\rho})= & \int_{V} d^{3} \mathbf{r}\left(1-\exp \left(i \sum_{m=1}^{6} \rho_{m} e_{m}(\mathbf{r})\right)\right) .
\end{aligned}
$$

Поскольку компоненты тензора деформации $e_{m}(\mathbf{r})$ в упруго анизотропном кристалле представляются численными массивами, построение функции распределения случайных деформаций выполняется численно. Выражение (5) для любых пар симметризованных компонент тензора деформации после интегрирования по модулю вектора $\mathbf{r}$ в функции $J(\boldsymbol{\rho})$ принимает вид

$$
\begin{aligned}
& g\left(e_{1}, e_{2}\right)=\frac{1}{(2 \pi)^{2}} \int_{-\infty}^{\infty} d \rho_{1} \int_{-\infty}^{\infty} d \rho_{2} \\
& \times \exp \left(-i \sum_{m=1}^{2}\left\{\rho_{m} e_{m}+\xi \int_{0}^{\pi} \sin \theta d \theta \int_{0}^{2 \pi} d \varphi\left|\rho_{m} q_{m}(\theta, \varphi)\right|\right\}\right),
\end{aligned}
$$

где $\xi=|\Omega| C_{d} / 48 \pi$ - единственный параметр, определяемый концентрацией и типом дефектов в конкретном кристалле, и интеграл в показателе экспоненты берется по сфере единичного радиуса. Анализ результатов численных расчетов функций распределения (7) для кристаллов различной структуры показал, что двумерная функция распределения может быть записана в виде обобщенной функции Лоренца

$$
g\left(e_{1}, e_{2}\right)=\frac{\xi \gamma v}{2 \pi}\left(\tilde{e}_{1}^{2}+v^{2} \tilde{e}_{2}^{2}+\xi^{2} \gamma^{2}\right)^{-3 / 2}
$$

Линейные преобразования $\tilde{e}_{1}=\cos \psi e_{1}+\sin \psi e_{2}, \tilde{e}_{2}=$ $=-\sin \psi e_{1}+\cos \psi e_{2}$ определяют направления главных осей эквипотенциальной поверхности распределения. Для рассмотренных в настоящей работе тетрагональных и тригональных кристаллов мы построили функции распределения (8) пар компонент $e(\Gamma, \lambda)$ тензора деформации, преобразующихся по одному и тому же неприводимому представлению. Соответствующие параметры $\gamma_{\Gamma}, v_{\Gamma}$ и $\psi_{\Gamma}$ функций распределения (8) различаются по индексу Г. В качестве примера на рис. 2 представлены функции распределения полносимметричных и ромбических деформаций в кристалле $\mathrm{LiLuF}_{4}$.

Обобщение функций рапределения (8) на случай шестимерного пространства компонент тензора деформа- 

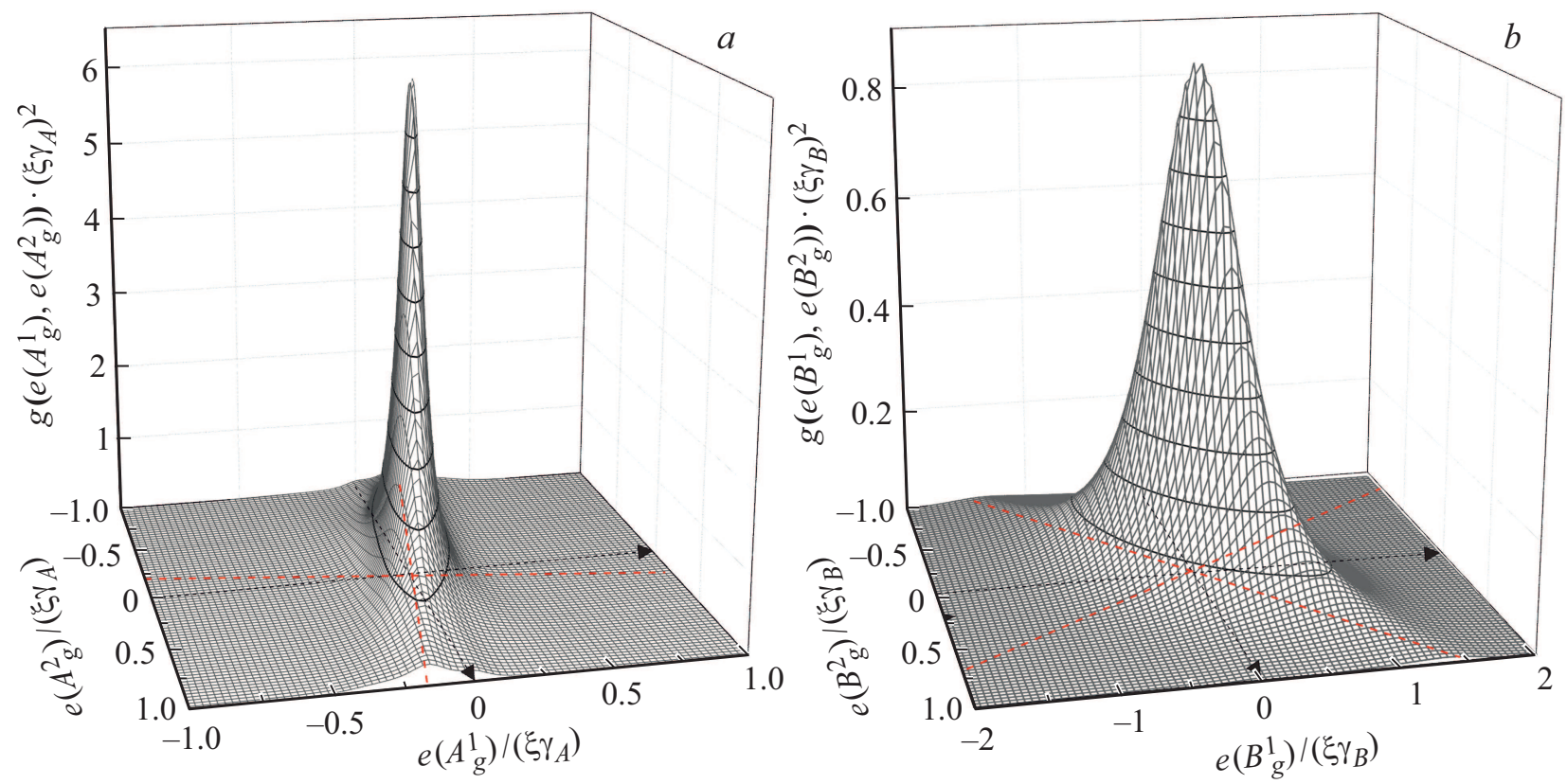

Рис. 2. Функции распределения случайных деформаций $g\left(e\left(\Gamma^{1}\right), e\left(\Gamma^{2}\right)\right) \cdot\left(\xi \gamma_{\Gamma}\right)^{2},\left(\right.$ слева $\Gamma=A_{g}$, справа $\left.-B_{g}\right)$, индуцированных точечными дефектами в кристалле $\mathrm{LiLuF}_{4}$. Штриховыми линиями обозначены главные оси распределения.

ции в кристалле $\mathrm{LiLuF}_{4}$ (см. [6]) имеет вид

$$
\begin{aligned}
g(\mathbf{e})= & \frac{15 \xi v_{A} \nu_{B}}{8 \pi^{3} \gamma_{A}^{2} \gamma_{B}^{2} \gamma_{E}^{2}}\left(\left(v_{A}^{2} \tilde{e}_{1}^{2}+\tilde{e}_{2}^{2}\right) / \gamma_{A}^{2}+\left(v_{B}^{2} \tilde{e}_{3}^{2}+\tilde{e}_{4}^{2}\right) / \gamma_{B}^{2}\right. \\
& \left.+\left(\tilde{e}_{5}^{2}+\tilde{e}_{6}^{2}\right) / \gamma_{E}^{2}+\xi^{2}\right)^{-7 / 2} .
\end{aligned}
$$

Для кристалла $\mathrm{LaAlO}_{3}$

$$
\begin{aligned}
g(\mathbf{e})= & \frac{15 \xi v_{A}}{8 \pi^{3} \gamma_{A}^{2} \gamma_{E^{1}}^{2} \gamma_{E^{2}}^{2}}\left(\left(v_{A}^{2} \tilde{e}_{1}^{2}+\tilde{e}_{2}^{2}\right) / \gamma_{A}^{2}+\left(\tilde{e}_{3}^{2}+\tilde{e}_{4}^{2}\right) / \gamma_{E^{1}}^{2}\right. \\
& \left.+\left(\tilde{e}_{5}^{2}+\tilde{e}_{6}^{2}\right) / \gamma_{E^{2}}^{2}+\xi^{2}\right)^{-7 / 2} .
\end{aligned}
$$

Значения параметров функций распределения (9) и (10) приведены в таблице. В предельном случае упруго изотропного континуума $v_{\Gamma}=1, \psi_{\Gamma}=0$, параметр $\gamma_{\Gamma}=16 \pi^{2}(1+\sigma) / 9(1-\sigma)$ не зависит от НП Г и определяется только коэффициентом Пуассона $\sigma$.

В следующем разделе функция распределения случайных деформаций (9) используется в моделировании тонкой структуры линии синглет-дублетного перехода в кристалле $\mathrm{LiLuF}_{4}: \mathrm{Pr}^{3+}$.

Параметры функций распределения случайных деформаций (9), (10) для кристаллов $\mathrm{LiLuF}_{4}$ и $\mathrm{LaAlO}_{3}$

\begin{tabular}{c|c|c|c|c|c|c}
\hline Параметр & \multicolumn{3}{|c|}{$\mathrm{LiLuF}_{4}$} & \multicolumn{3}{c}{$\mathrm{LaAlO}_{3}$} \\
\hline$\Gamma$ & $A$ & $B$ & $E$ & $A$ & $E^{1}$ & $E^{2}$ \\
\hline$\gamma_{\Gamma}$ & 30.3 & 54.0 & 30.4 & 26.2 & 30.7 & 31.2 \\
$\nu_{\Gamma}$ & 5.26 & 2.32 & 1 & 5.88 & 1 & 1 \\
$\psi_{\Gamma}$ & $9.2^{\circ}$ & $-35^{\circ}$ & 0 & $7.4^{\circ}$ & 0 & 0
\end{tabular}

\section{4. Форма линии синглет-дублетного перехода в кристалле LiLuF 4 : $\mathrm{Pr}^{3+}$}

Поляризованные спектры поглощения монокристалла $\mathrm{LiLuF}_{4}$, активированного ионами $\mathrm{Pr}^{3+}(0.1$ at.\%), были измерены при температурах $5-300 \mathrm{~K}$ фурье-спектрометром высокого разрешения (до $0.008 \mathrm{~cm}^{-1}$ ) BRUKER IFS 125, оснащенным криостатом замкнутого цикла Cryomech ST 403 и охлаждаемым жидким азотом InSb детектором. Кристаллическое поле, действующее на ионы $\mathrm{Pr}^{3+}$, замещающие ионы $\mathrm{Lu}^{3+}$ в позициях с локальной симметрией $S_{4}$, расщепляет электронные мультиплеты $4 f^{2}$-оболочки на синглеты $\Gamma_{1}$ и $\Gamma_{2}$ и дублеты $\Gamma_{34}$ $\left(\Gamma_{p}-\right.$ НП группы $\left.S_{4}\right)$. На одной из наиболее узких линий в ИК области спектра, отвечающей переходу из основного состояния $\Gamma_{2}\left({ }^{3} H_{4}\right)$ на дублет $\Gamma_{34}\left({ }^{3} H_{5}\right)$ (см. рис. 3), наблюдалась дублетная структура, показанная на рис. 4.

Спектр энергий электронно-ядерных состояний иона ${ }^{141} \mathrm{Pr}^{3+}$ (существует единственный стабильный изотоп празеодима с ядерным спином $I=5 / 2$ ) в кристалле описывается гамильтонианом

$$
H=H_{F I}+H_{\mathrm{CF}}+H_{\mathrm{HFM}}+H_{\mathrm{el-def}},
$$

где $H_{F I}$ - энергия свободного иона [17], $H_{\mathrm{CF}}-$ энергия взаимодействия иона с кристаллическим полем,

$$
\begin{aligned}
H_{\mathrm{CF}}= & \sum\left(B_{2}^{0} O_{2}^{0}+B_{4}^{0} O_{4}^{0}+B_{6}^{0} O_{6}^{0}+B_{4}^{4} O_{4}^{4}\right. \\
& \left.+B_{4}^{-4} O_{4}^{-4}+B_{6}^{4} O_{6}^{4}+B_{6}^{-4} O_{6}^{-4}\right),
\end{aligned}
$$


$H_{\mathrm{HFM}}$ - энергия магнитного сверхтонкого взаимодействия,

$$
\begin{aligned}
H_{\mathrm{HFM}} & =\mu_{B} \gamma_{N} \hbar\left\langle\frac{1}{r^{3}}\right\rangle_{4 f} \sum\left\{2 I l+O_{2}^{0}\left(3 s_{z} I_{z}-s I\right)\right. \\
& +3 O_{2}^{2}\left(s_{x} I_{x}-s_{y} I_{y}\right)+3 O_{2}^{-2}\left(s_{x} I_{y}+s_{y} I_{x}\right) \\
& \left.+6 O_{2}^{1}\left(s_{x} I_{z}+s_{z} I_{x}\right)+6 O_{2}^{-1}\left(s_{z} I_{y}+s_{y} I_{z}\right)\right\},
\end{aligned}
$$

$H_{\mathrm{el}-\mathrm{def}}$ - электрон-деформационное взаимодействие,

$$
\begin{gathered}
H_{\mathrm{el}-\mathrm{def}}=\sum_{\Gamma \lambda} V(\Gamma, \lambda) e(\Gamma, \lambda), \\
V(\Gamma, \lambda)=\sum_{p k} \sum b_{p}^{k}(\Gamma, \lambda) O_{p}^{k} .
\end{gathered}
$$

В формулах (12)-(14) символ $\sum$ означает суммирование по $4 f$-электронам с орбитальными и спиновыми моментами $l$ и $s, \gamma_{N} / 2 \pi=13.05 \mathrm{~Hz} / \mathrm{T}-$ ядерное гиромагнитное отношение [18], $\mu_{B}-$ магнетон Бора, $\left\langle 1 / r^{3}\right\rangle_{4 f}=5$ a.u. [19]. Операторы $O_{p}^{k}-$ линейные комбинации одноэлектронных сферических тензорных операторов, определенные в [20] (в пространстве состояний мультиплета с полным моментом $J$ операторы $O_{p}^{k}$ совпадают с операторами Стивенса). В численных расчетах собственных значений оператора $H^{\prime}=H_{F I}+H_{\mathrm{CF}}+H_{\mathrm{HFM}}$, действующего в пространстве 546 электронно-ядерных состояний $4 f^{2}$-оболочки, были использованы параметры гамильтониана свободного иона $H_{F I}$ из работы [21]. Параметры кристаллического поля $B_{2}^{0}=216.5, B_{4}^{0}=-130$, $B_{6}^{0}=-2.94, \quad B_{4}^{4}=-1127, \quad B_{4}^{-4}=-849, \quad B_{6}^{4}=-635$, $B_{6}^{-4}=-428 \mathrm{~cm}^{-1}$ были определены моделированием результатов измерений штарковской структуры мультиплетов ${ }^{3} H_{4,5,6},{ }^{3} F_{2,3,4},{ }^{3} P_{0,1,2}$. Параметры оператора электрон-деформационного взаимодействия $b_{p}^{k}(\Gamma, \lambda)$ были вычислены в рамках модели обменных зарядов [22] и затем скорректированы по результатам пьезоспектроскопического исследования кристалла $\mathrm{LiLuF}_{4}: \mathrm{Tm}^{3+}[23]$.

В первом приближении по $H_{\mathrm{HFM}}$ сверхтонкое взаимодействие дает нулевой вклад в энергию синглетов $\Gamma_{1}$ и $\Gamma_{2}$, а дублеты $\Gamma_{34}$ расщепляются на шесть эквидистантных электронно-ядерных дублетов с энергиями $A M m$ (где $M= \pm 1, m= \pm 1 / 2, \pm 3 / 2, \pm 5 / 2-$ проекции спина ядра на ось симметрии $c, A-$ интервал сверхтонкой структуры). В частности, вычисленные интервалы сверхтонкой структуры дублета $\Gamma_{34}\left({ }^{3} H_{5}\right)$ равны $0.0154 \pm 0.0015 \mathrm{~cm}^{-1}$ (эквидистантность нарушается вследствие смешивания волновых функций штарковских подуровней мультиплетов сверхтонким взаимодействием). Однако вместо квази-эквидистантной шестикомпонентной сверхтонкой структуры линия синглет-дублетного перехода $\Gamma_{2}\left({ }^{3} H_{4}\right) \rightarrow \Gamma_{34}\left({ }^{3} H_{5}\right)$

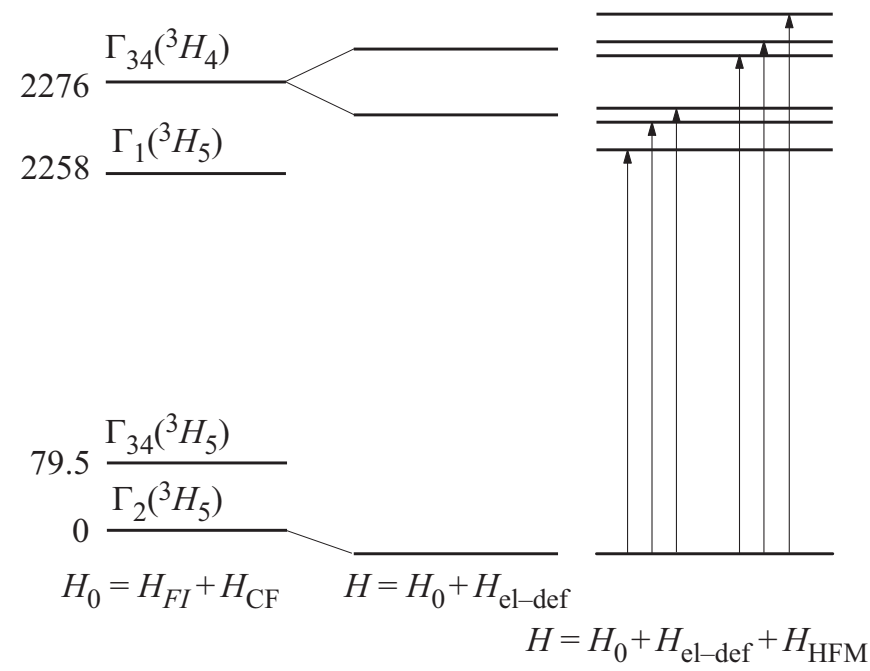

Рис. 3. Схема уровней энергии $\left(\mathrm{cm}^{-1}\right)$ иона $\operatorname{Pr}^{3+}$ в кристалле $\mathrm{LiLuF}_{4}$.

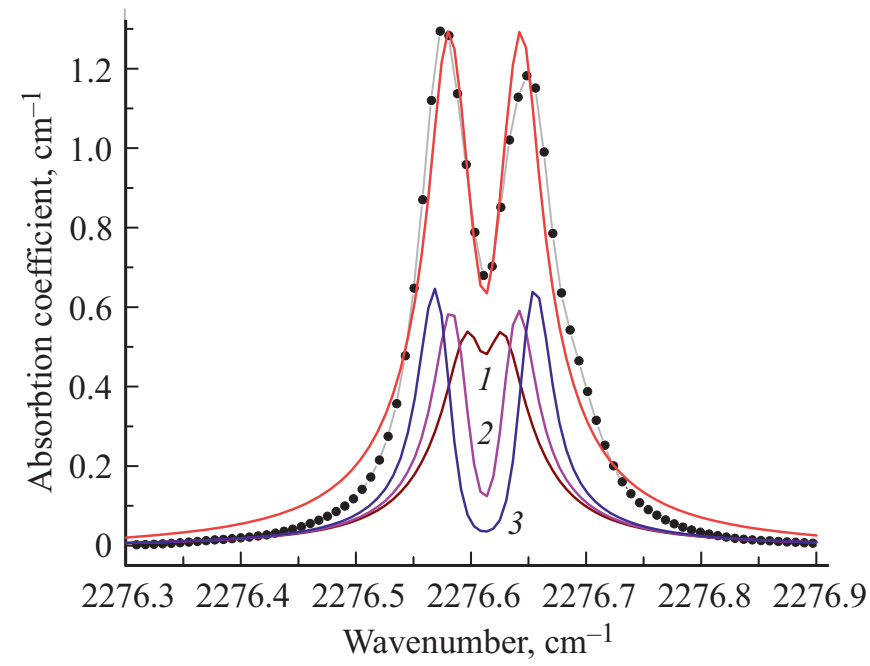

Рис. 4. Форма линии (символы - эксперимент, сплошные линии - расчет), соответствующей переходам $\Gamma_{2}\left({ }^{3} H_{4}\right) \rightarrow \Gamma_{34}\left({ }^{3} H_{5}\right)$ в $\sigma$-поляризации $(\mathbf{k} \perp \mathbf{c}, \mathbf{E} \perp c, \mathbf{H} \| c)$ ионов $\operatorname{Pr}^{3+}$ в кристалле $\mathrm{LiLuF}_{4}$. Линии 1,2 и 3 спектральные огибающие переходов между электронноядерными состояниями с проекциями спина ядра $|m|=1 / 2$, $3 / 2$ и $5 / 2$ соответственно.

содержит лишь один провал с шириной, сравнимой с полной шириной вычисленной сверхтонкой структуры (5A) дублета (см. рис. 4).

Электрон-деформационное взаимодействие мы рассматриваем в первом приближении теории возмущений. Полносимметричные деформации $e\left(A_{g}^{1}\right)$ и $e\left(A_{g}^{2}\right)$ смещают дублет $\Gamma_{34}\left({ }^{3} H_{5}\right)$ относительно синглета $\Gamma_{2}\left({ }^{3} H_{4}\right)$, ромбические деформации $e\left(B_{g}^{1}\right)$ и $e\left(B_{g}^{2}\right)$ индуцируют дополнительное расталкивание сверхтонких подуровней дублета с одинаковыми величинами проекций $m$ ядерного спина на ось $c$ (см. рис. 3). Сдвиги компонент сверхтонкой структуры относительно центра линии при 
фиксированных деформациях принимают вид

$$
\begin{aligned}
\varepsilon_{|m|}^{( \pm)}= & v\left(A_{g}^{1}\right) e\left(A_{g}^{1}\right)+v\left(A_{g}^{2}\right) e\left(A_{g}^{2}\right) \\
& \pm \sqrt{\Delta_{|m|}^{2}+\left|v\left(B_{g}^{1}\right) e\left(B_{g}^{1}\right)+v\left(B_{g}^{2}\right) e\left(B_{g}^{2}\right)\right|^{2}}
\end{aligned}
$$

где $\quad \Delta_{|m|}=A|m|, \quad v\left(A_{g}^{1}\right)=-559 \mathrm{~cm}^{-1} \quad$ и $\quad v\left(A_{g}^{2}\right)=$ $=-443 \mathrm{~cm}^{-1}-$ разности диагональных матричных элементов введенных в (14) операторов $V\left(A_{g}^{n}\right)$ на электронных волновых функциях дублета $\Gamma_{34}\left({ }^{3} H_{5}\right)$ и синглета $\Gamma_{2}\left({ }^{3} H_{4}\right), \quad v\left(B_{g}^{1}\right)=-553+446 i \quad$ и $v\left(B_{g}^{2}\right)=-375+478 i \mathrm{~cm}^{-1}-$ недиагональные матричные элементы операторов $V\left(B_{g}^{n}\right)(n=1,2)$ на электронных волновых функциях дублета.

Спектральную огибающую $I(\omega)$ линии синглетдублетного перехода получаем усреднением по распределению случайных деформаций (9) суммы форм-функций компонент сверхтонкой структуры:

$$
\begin{gathered}
I(\omega) \propto \int g(\mathbf{e}) \sum_{m=\frac{1}{2}, \frac{3}{5}, \frac{5}{2}}\left[I_{0}\left(\hbar\left(\omega-\omega_{0}\right)-\varepsilon_{m}^{(+)}(\mathbf{e})\right)\right. \\
\left.\quad+I_{0}\left(\hbar\left(\omega-\omega_{0}\right)-\varepsilon_{m}^{(-)}(\mathbf{e})\right)\right] d \mathbf{e},
\end{gathered}
$$

где частота $\omega_{0}$ соответствует центру линии, форм-функцию аппроксимируем распределением Гаусса $I_{0}(x)=\left(2 \pi \Delta^{2}\right)^{-1 / 2} \exp \left(-x^{2} / 2 \Delta^{2}\right)$ с дисперсией $\Delta^{2}$.

Ширина функции распределения случайных деформаций $\xi=0.75 \cdot 10^{-6}$ в исследованном кристалле $\mathrm{LiLuF}_{4}$ и параметр форм-функции $\Delta=0.008 \mathrm{~cm}^{-1}$ найдены из сопоставления вычисленной спектральной огибающей перехода $\Gamma_{2}\left({ }^{3} H_{4}\right) \rightarrow \Gamma_{34}\left({ }^{3} H_{5}\right)$ с измеренным спектром. Вычисленные распределения интенсивностей переходов между электронно-ядерными состояниями синглета и дублета с различными проекциями $m$ спина ядра представлены на рис. 4. Оценка силы дефекта примесного иона празеодима с использованием полученной ширины распределения случайных деформаций $\xi$ $|\Omega|=48 \pi \xi V / 2 x=0.0565 \cdot V=7.8 \AA^{3}(V$ - объем элементарной ячейки, содержащей два иона лютеция, $x=0.001-$ номинальная концентрация примесных ионов) сопоставима с возможным изменением объема ячейки $4 \pi\left(R_{\mathrm{Pr}}^{3}-R_{\mathrm{Lu}}^{3}\right) / 3=2.1 \AA^{3}$, обусловленным различием ионных радиусов празеодима $\left(R_{\operatorname{Pr}}=1.126 \AA\right)$ и лютеция $\left(R_{\mathrm{Lu}}=0.977 \AA\right)$ [24]. Таким образом, можно сделать вывод о формировании поля случайных деформаций как собственными дефектами кристаллической решетки, так и примесными ионами.

\section{5. Заключение}

Построена функция распределения для шести компонент тензора деформаций, индуцированных точечными дефектами в упруго анизотропных кристаллах, получены параметры функций распределения деформаций в кристаллах $\mathrm{LiLuF}_{4}$ и $\mathrm{LaAlO}_{3}$. В оптическом спектре поглощения высокого разрешения кристалла $\mathrm{LiLuF}_{4}$, активированного ионами $\operatorname{Pr}^{3+}(0.1$ ат.\%), зарегистрирована линия синглет-дублетного перехода ионов празеодима в тетрагональном кристаллическом поле со специфической дублетной структурой вместо ожидаемой шестикомпонентной сверхтонкой структуры (спин ядра ${ }^{141} \mathrm{Pr}$ $I=5 / 2$ ). Моделирование спектральных огибающих компонент сверхтонкой структуры с учетом взаимодействия ионов $\mathrm{Pr}^{3+}$ со случайными деформациями решетки дало возможность успешно воспроизвести измеренную форму линии.

Представленный анализ формы тонкой структуры линии синглет-дублетного перехода демонстрирует возможность восстановления сверхтонкой структуры некрамерсовского дублета, скрытой неоднородным деформационным уширением, при помощи теории, развитой в настоящей работе. Моделирование оптических спектров высокого разрешения можно использовать для анализа качества оптических материалов.

\section{Список литературы}

[1] R. Kolesov, K. Xia, R. Reuter, R. Stöhr, A. Zappe, J. Meijer, P.R. Hemmer, J. Wrachtrup. Nature Commun. 3, 1029 (2012).

[2] T. Zhong, J.M. Kindem, E. Miyazono, A. Faraon. Nature Commun. 6, 8206 (2015).

[3] A.M. Stoneham. Rev. Mod. Phys. 41, 82 (1969).

[4] S.A. Klimin, D.S. Pytalev, M.N. Popova, B.Z. Malkin, M.N. Vanyunin, S.L. Korableva. Phys. Rev. B 81, 045113 (2010).

[5] B.Z. Malkin, D.S. Pytalev, M.N. Popova, E.I. Baibekov, M.L. Falin, K.I. Gerasimov, N.M. Khaidukov. Phys. Rev. B 86, 134110 (2012).

[6] B.Z. Malkin, N.M. Abishev, E.I. Baibekov, D.S. Pytalev, K.N. Boldyrev, M.N. Popova, M. Battinelli. Phys. Rev. B 96, 014116 (2017).

[7] K.N. Boldyrev, P. Dereń, M.N. Popova. EPJ Web Conf. 132, 03004 (2017).

[8] Л.Д. Ландау, Е.М. Лифшиц. Теория упругости. Наука, М. (1965). 204 c.

[9] J.D. Eshelby. Solid State Phys. 3, 79 (1956).

[10] И.М. Лифшиц, Л.Н. Розенцвейг. ЖЭТФ 17, 9, 783 (1947).

[11] А.М. Косевич. Основы механики кристаллической решетки. Наука, М. (1972). 280 с.

[12] D.M. Barnett. Phys. Status Solidi B 49, 741 (1972).

[13] L.J. Gray, D. Ghosh, T. Kaplan. Comput. Mech. 17, 255 (1996).

[14] F.C. Buroni, A. Sáez. Proc. R. Soc. A 466, 515 (2010).

[15] С.А. Альтшулер и др. Магнитоупругие явления в двойных фторидах редких земель. В сб.: Парамагнитный резонанс. Изд-во Казанского университета (1984). 29 с.

[16] M.A. Carpenter, S.V. Sinogeikin, J.D. Bass, D.L. Lakshtanov. J. Phys.: Condens. Matter 22, 035403 (2010).

[17] W.T. Carnall, G.L. Goodman, K. Rajnak, R.S. Rana. J. Chem. Phys. 90, 3443 (1989). 
[18] K.K. Sharma, L.E. Erickson. J. Phys. C 14, 1329 (1981).

[19] A. Abragam, B. Bleaney. Electron paramagnetic resonance of transition ions. Clarendon Press, Oxford (1970).

[20] V.V. Klekovkina, A.R. Zakirov, B.Z. Malkin, L.A. Kasatkina. J. Phys.: Conf. Ser. 324, 012036 (2011).

[21] M.J. Lee, M.F. Reid, M.D. Faucher, G.W. Burdick. J. Alloys Comp. 323\&324, 636 (2001).

[22] B.Z. Malkin. Crystal field and electron-phonon interaction in rare-earth ionic paramagnets. In: Spectroscopy of solids containing rare-earth ions/ Ed. A.A. Kaplyanskii, R.M. Macfarlane. Elsevier Science Publishers, Amsterdam (1987). Ch. 2. P. $13-49$.

[23] А.В. Винокуров, Б.З. Малкин, А.И. Поминов, А.Л. Столов. ФTT 30, 3426 (1988).

[24] R.D. Shannon. Acta Cryst. A 32, 751 (1976).

Редактор Е.Ю. Флегонтова 